\title{
Robust nanosized transistor effect in fullerene-tube heterostructure
}

\author{
Per Hyldgaard and Bengt I. Lundqvist \\ Department of Applied Physics, Chalmers University of Technology and Göteborg University, \\ S-412 96 Göteborg, Sweden
}

(October 25, 2002)

\begin{abstract}
A fullerene-tube heterostructure should produce a compact resonanttunneling system, when a nanometer section of non-conducting nanotube separates a pair of metallic-nanotubes leads. Adding a set of metal contacts and gates completes our proposal for a robust current switch and transistor with a nanosized feature size. The electrostatic gates provide a crisp resonance-level control for the orbitals trapped in the nonconducting barrier and can thus selectively enable/inhibit a strong resonant-tunneling current. A conserving Green function study documents that the transistor effect is robust at room temperature and in the presence of a strong inelastic scattering.

1999 PACS number: 71.20.Tx,72.80.Rj,73.61.Wp,85.30.Tv
\end{abstract}

Typeset using REVTEX 
The accelerating rate $^{1}$ of electronics miniaturization cannot be indefinitely sustained within the traditional semiconductor technology. For alternatives the molecular-bond subnanometer length sets an ultimate limit, but there is still plenty of room. ${ }^{2}$ Fullerene nanotubes ${ }^{3,4}$ offer fascinating candidates for a nanosized molecular-electronics program, as the nature of their conduction is controlled by the local chirality and radius. ${ }^{5,6}$ Present room-temperature fullerene transistors ${ }^{7}$ use semiconducting nanotubes adsorbed between metal contacts and across a gate to permit electrostatic field-effect control of the semi-classical transport. ${ }^{8}$ Unfortunately, the semiconductor nanotubes offer little confinement of the necessary electrostatic field while the semi-classical operation imposes a mesoscopic characteristic feature size, $L_{f} \gtrsim 0.1 \mu \mathrm{m}$. We show that electrostatic fields can control also nanoscale electronics devices and that an intra-nanotube heterostructure with a feature size $L_{f} \sim 10 \mathrm{~nm}$ should be able to realize a current-switch and transistor effect with good electrostatic confinement. In short, our transistor device is of a field-effect design ${ }^{7,8}$ but replaces the transport operation by a necessary quantum-mechanical one: nonequilibrium resonant tunneling. ${ }^{9}$ A current-conserving transport calculation ${ }^{10-12}$ documents device robustness, even at room temperature.

Figure 1 summarizes the quantum-mechanical transistor operation, which rests on the following ideas: (1) A nanoscale section of nonconducting-fullerene transport barrier (central honeycomb region) separates a pair of metallic nanowires (grey horizontal tubes, ' $L$ ' and ' $R$ '), which form leads to the source and drain contacts (top triangles); These nanotube sections of different chirality are joined by structural (heptagon/pentagon) defects ${ }^{13}$ like in the nanotube-heterojunction diode ${ }^{14}$; The contacts, e.g., traditional scanning-tunneling microscope tips, sustain the nanowire leads at different chemical potentials, $\mu_{\mathrm{L}}=\mu_{\mathrm{R}}+e V_{\text {bias }}$, by the moderate applied bias, $V_{\text {bias }} \sim 150 \mathrm{mV}$. (2) Transport then requires tunneling through the well-resolved lowest unoccupied molecular orbital $\Psi_{\text {orb }}$ (left panel) trapped in the barrier region; This resonance connects to the surrounding leads by rates $\Gamma_{\mathrm{L} / \mathrm{R}} \sim 10 \mathrm{meV}$. (3) $\mathrm{A}$ close gate (bottom triangle), at voltage $\Phi_{\text {gate }}$, controls (right panel) the drain-to-source load current $J$ by adjusting the resonance energy position $E_{\text {orb }}\left(\Phi_{\text {gate }}\right)-(3 a)$ At $\Phi_{\text {gate }} \sim 0$ 
the molecular energy gap, ${ }^{15} E_{g} \sim 1 \mathrm{eV}$ (dashed box), forces the resonance (dashed line) far above the chemical potentials $\mu_{\mathrm{L} / \mathrm{R}}$ and ensures $J \rightarrow 0$. However, (3b) $\Phi_{\text {gate }} \sim 2 \mathrm{~V}$ suppresses the barrier-region electron potential (solid curve) and adjusts $E_{\text {orb }}$ (solid line) to enable tunneling (arrows). (4) The resonance at $E_{\text {orb }}$ then sustains a nonequilibrium electron occupation $\left\langle n_{\text {orb }}\right\rangle \approx 1$ and carries a strong, temperature- and scattering-insensitive load current, ${ }^{11,12}$

$$
J \leq J_{\mathrm{RT}} \equiv\left(\frac{e}{\hbar}\right) \frac{4 \Gamma_{\mathrm{L}} \Gamma_{\mathrm{R}}}{\Gamma_{\mathrm{L}}+\Gamma_{\mathrm{R}}} \sim 5 \mu \mathrm{A}
$$

(5) In contrast, the gate-to-source input current remains insignificant, $J_{\text {in }} \approx J_{\mathrm{V}}\left\langle n_{\text {orb }}\right\rangle / 2<$ $J_{\mathrm{V}} \ll 1 \mathrm{nA}$, at the illustrated gate-to-barrier separation; This current is defined by the very small gate-to-barrier vacuum tunneling ${ }^{16,17} J_{\mathrm{V}}$. Thus, (6) the nanotube structure produces a large-amplification transistor effect, $J \propto J_{\text {in }}$, controlled by the gate voltage $\Phi_{\text {gate }}$. The scale of the indicated contact separation, $L_{f} \sim 10 \mathrm{~nm}$, defines the characteristic transistor feature size in the metal-contact realization.

The prospect to materialize our proposed transistor is promising. A doubleheterojunction nanotube transistor, Fig. 1, with a larger size has already been produced ${ }^{18}$, like the single-heterojunction current-rectifying diode, ${ }^{14}$ and could also be engineered. ${ }^{19} A$ nonrobust, ultra-low temperature $T \sim 50 \mathrm{mK}$, resonant-tunneling transistor effect has already been observed and used to document metallic conduction in the nanotube quantum wires. $^{4,20}$ There the total wire length $L_{\text {wire }}$ defines a level spacing $1 \mathrm{meV} \gg \delta E_{\text {wire }} \propto 1 / L_{\text {wire }}$. For $e V_{\text {bias }}, T<\delta E_{\text {wire }}$, however, an electrostatically controlled resonant tunneling still probes the transport through a set of quantum-wire levels and subject to electron-electron interaction effects. ${ }^{20}$ The robust resonant-tunneling transistor effect should arise in the nanotube heterostructure, Fig. 1, as we substitute the barrier extension $L_{B}$ for $L_{\text {wire }}$, enhance the level spacing, and focus the transport onto well-resolved molecular levels, $E_{\text {orb }}$. The present production of double-junction transistors ${ }^{18}$ utilizes a large number of nanotube samples that are distributed onto metal contacts and scanned, e.g., by transmission electron microscopy, for the kinks, ${ }^{14,18}$ which identifies the individual heterojunctions. ${ }^{13,14}$ Our need for selection 
and wiring of samples with a nanoscale kink separation calls for alternative methods for fabrication. The engineering control proposed recently ${ }^{19}$ could be one possibility.

The choice of metallic-nanotube leads should permit a natural confinement of the electrostatic fields, i.e. electronics packaging, and a robust well-characterized nanosized transistor operation. We have theoretically checked that the electrostatic control should allow transistor operation even with such a close (nanoscale) proximity of metal contacts and metallicnanotube leads. In one way our structure resembles the recently investigated intra-nanotube quantum dot: ${ }^{21}$ a set of resonance orbitals are trapped. However, we propose the opposite heterostructure, where, for example, a pair of $(5,5)$ 'armchair' metallic leads surround a central (semi-)nonconducting barrier region of chirality $(6,4)$; Fig. 1. The metallic leads then confine the electrostatic-gate effect to the barrier region, extending $L_{B} \approx 3 \mathrm{~nm}$, and should realize a strong automatic packaging. They should also ensure a strong current injection and similar gate control for all nanoscale contact separations (feature sizes) $L_{f} \gtrsim 3 L_{B} \sim 10 \mathrm{~nm}$. As documented below, the resulting well-characterized resonant-tunneling transport is insensitive to the strong scattering that a finite temperature and applied bias can induce. ${ }^{11,12,22}$

Figure 2 documents the current-switch effect, $J \equiv J\left(\Phi_{\text {gate }}\right)$ (top panel), and the transistor effect, $J \propto J_{\text {in }}$ (bottom panel). Both types of device operation result from gate-voltage $\left(\Phi_{\text {gate }}\right)$ adjustment of the lowest barrier-resonance level $E_{\text {orb. }}$. To quantify this crucially important current-switch/transistor gate control, $E_{\text {orb }}=E_{\text {orb }}\left(\Phi_{\text {gate }}\right)$, we first present an estimate of the electrostatic-field effects for the nonconducting barrier section, when surrounded by metallic leads (Fig. 1).

The general electrodynamic response of the molecular structure is given through a consistent calculation of the electron-density variation $n(r)$, and to a high degree of accuracy, the local frequency-dependent dielectric response, ${ }^{23,24}$

$$
\varepsilon(n(r), \omega)=1-\frac{4 \pi e^{2} n(r) / m}{\omega^{2}+\omega_{0}^{0}},
$$

where $m_{e}$ denotes the electron mass. A calculation of the overall static molecular polarization establishes a sensible value for the frequency cut off $\omega_{0}$ and thus permits a simple, yet 
accurate description of even the mutual molecular van der Waals interaction, ${ }^{24}$ which arises from the dynamical polarization response. The simple Drude-like response (2) and a separate density-functional calculation ${ }^{25}$ of the nanotube electron density $n(r)$ are used to characterize also the nanotube electrostatic field response subject to the boundary conditions imposed by the metallic leads. In our sample realization (Fig. 1) the application of the external gate voltage $\Phi_{\text {gate }}$ is found to cause a reduction $e \delta \Phi_{\mathrm{B}} \approx-e \Phi_{\text {gate }} / 3$ of the potential that define the electron dynamics inside the nonconducting nanotube barrier section. A gate voltage $\Phi_{\text {gate }} \approx 2 \mathrm{~V}$ ensures potential adjustments which exceed half the molecular-barrier energy gap, $e \delta \Phi_{\mathrm{B}} \gtrsim E_{g} / 2$, and thus enables the resonant-tunneling transistor current (Fig. 1, right panel).

A combination of an effective nanotube tight-binding Hamiltonian model ${ }^{5}$ and a nonequilibrium Green function description ${ }^{10-12}$ details this electrostatic-gate control of the transistor current. The 'armchair' metallic-nanotube leads comprise rings of carbon atoms repeated every distance $a=\sqrt{3} d$, where $d=0.142 \mathrm{~nm}$ denotes the carbon-carbon bond length. The pair $\eta=1,2$ of metallic-nanotube transport channels is described ${ }^{5}$ by associating each such carbon ring with two transport sites connected by effective overlap integrals $W_{\eta}=(-1)^{\eta} 2.66 \mathrm{eV}$, respectively. We use a similar description for the (unoccupied) states in the central barrier region and choose transport parameters consistent with both recent (zero-gate) electronic-structure calculations ${ }^{13,21}$ and our estimate for the electrostatic-field effects. A set of sites (here thirteen), enumerated along the wire, $n_{\mathrm{L}} \leq n \leq n_{\mathrm{R}}$, describe the molecular-barrier dynamics by an overlap integral $t=3\left|W_{\eta}\right| / 4-E_{g} / 8 \approx 2 \mathrm{eV}$ and site energies $E_{n}$. The gate-induced change in barrier potential, $e \delta \Phi_{\mathrm{B}} \propto \Phi_{\text {gate }}$, causes a direct modification of these barrier site energies, $E_{n}\left(\Phi_{\text {gate }}\right)$; An exact diagonalization of the barrier tight-binding dynamics establishes the gate control of the barrier resonances $\left(E_{\text {orb }}\right)$ and the associated wavefunctions $\left(\Psi_{\text {orb }}(n)\right)$. Tunneling-escape rates, $\Gamma_{\mathrm{L} / \mathrm{R}}(E) \approx t\left|\Psi_{\text {orb }}\left(n_{\mathrm{L} / \mathrm{R}}\right)\right|^{2} \sum_{\eta} v_{\mathrm{L} / \mathrm{R} \eta}(E)$, then describe the coupling between barrier and surrounding leads. Here $v_{\mathrm{L} / \mathrm{R}}(E)$ denotes a dimensionless measure of the lead electron velocity at energy $E$ in channel $\eta$. The energy-dependence is weak, $v_{\mathrm{L} / \mathrm{R}, \eta}\left(E \approx E_{\text {orb }}\right) \approx \sqrt{3 / 4}$, 
and we may approximate $\Gamma_{\mathrm{L} / \mathrm{R}} \equiv \Gamma_{\mathrm{L} / \mathrm{R}}\left(E_{\mathrm{orb}}\right)$. The gate-induced wavefunction confinement (Fig. 1, left panel) causes a weak rate reduction to $\Gamma_{\mathrm{L} / \mathrm{R}} \approx 10 \mathrm{meV}$ at operating conditions, $e \delta \Phi_{\mathrm{B}} \gtrsim E_{g} / 2\left(\Phi_{\text {gate }} \approx 2 \mathrm{~V}\right)$; adjusting the diameter of the nanotube-barrier, and hence the molecular-energy gap $E_{g},{ }^{15}$ permits further control of the resonance width, $\Gamma \equiv \Gamma_{\mathrm{L}}+\Gamma_{\mathrm{R}}$. This width, estimated at $\Gamma \approx 20 \mathrm{meV}$, is used as a common scale to simplify discussions.

The insert panel summarizes our transistor electron-dynamics study and validates a single-resonance transport discussion of device robustness (below). The solid and dasheddotted curves detail the electrostatic control and report the calculated variations of $E_{\text {orb }}\left(\Phi_{\text {gate }}\right)$ and $E_{\text {orb }}^{*}\left(\Phi_{\text {gate }}\right)$, the lowest and second-lowest resonance-energy levels. The pair of dashed lines identify the zone $\left|\mu_{\mathrm{L}}-E_{\text {orb }}\right|<6 \Gamma$, which defines the proposed working conditions for the current-switch and transistor operation, $e V_{\text {bias }} \approx 150 \mathrm{meV}(\sim 6 \Gamma)$. This device-operation zone lies well within the resonance level separation, $E_{\text {orb }}^{(*)}-E_{\text {orb. }}$ Transport is thus focused onto the temperature- and scattering-insensitive ${ }^{12}$ single-resonance mechanism that we discuss below.

The top panel in Fig. 2 illustrates the resulting robust nanosized current-switch operation at a fixed applied bias, $e V_{\text {bias }}=6 \Gamma$. The solid curve shows the load-current estimate

$$
J\left(E_{\mathrm{orb}}\right) \approx J_{\mathrm{RT}} \sum_{X=\mathrm{L}, \mathrm{R}} S_{X} \arctan \left(\frac{\mu_{X}-E_{\mathrm{orb}}}{\Gamma}\right)
$$

which summarizes our interacting nonequilibrium Green-function- transport study ${ }^{10-12}$ with $S_{L}=+1 / \pi, S_{R}=-1 / \pi$, and a peak current value in Eq. (1). The implicit gate control (insert panel) adjusts $E_{\text {orb }}$ to enable the resonant-tunneling load current $J \sim J_{\mathrm{RT}}$ ('on') at

$$
\left(\mu_{\mathrm{L}}-E_{\text {orb }}\right) \gtrsim \Gamma \quad \text { and } \quad\left(E_{\text {orb }}-\mu_{\mathrm{R}}\right) \gtrsim \Gamma
$$

The resonant-tunneling current is disabled ('off') and small when either $\left(E_{\text {orb }}-\mu_{\mathrm{L}}\right) \gtrsim 6 \Gamma$ or $\left(\mu_{\mathrm{R}}-E_{\text {orb }}\right) \gtrsim 6 \Gamma$. Finally, the bottom panel documents the corresponding transistor operation, $J \propto J_{\text {in }}$, for which we predict a robust large amplification, $J_{\mathrm{RT}} / J_{\mathrm{V}}>10^{3}$.

Figure 2 also emphasizes that the proposed current-switch and transistor effects are robust, i.e. that they are both temperature- and scattering-insensitive. For example, the top 
panel contrasts the load current estimate (3) (solid curve) against a finite-temperature evaluation (dotted curve) and an interacting-transport calculation (dashed curve), in which we include a strong nonequilibrium coupling to a local phonon mode of energy $\Omega_{0}=60 \mathrm{meV}$. We choose this interaction as a representative illustration and exaggerate the electron-phonon interacting matrix element, $M_{\mathrm{e}-\mathrm{p}}=10 \mathrm{meV}$, to stress the robustness. Neither temperature nor this scattering cause any significant change in the transistor-load current, when the applied bias satisfies

$$
e V_{\text {bias }} \equiv \mu_{\mathrm{L}}-\mu_{\mathrm{R}} \gg \max [T, \Gamma]
$$

This important transistor robustness rests upon conservation rules and can be explained through a link ${ }^{12}$ between the nonequilibrium transport and the interacting resonant-level density of state (DOS) $N_{\text {orb }}(E)$. This proof of scattering insensitivity explains, at the same time, why a noninteracting (linear-response) formalism ${ }^{9,26}$ also can reproduce the transistor estimate (3), albeit not rationalize the device robustness. The noninteracting resonanceorbital DOS is specified by

$$
N_{\text {orb }}^{0}(E)=\frac{\Gamma / \pi}{\left(E-E_{\text {orb }}\right)^{2}+\Gamma^{2}}
$$

We emphasize the use of a current-conserving ${ }^{10}$ diagrammatic technique $^{11,12}$ to establish the interaction change, $\delta N_{\text {orb }}(E)=N_{\text {orb }}(E)-N_{\text {orb }}^{0}(E)$, in the DOS. The interacting current may then be expressed as ${ }^{11,12}$

$$
J \approx J_{\mathrm{RT}} \int d E N_{\mathrm{orb}}(E)\left[f_{\mathrm{L}}(E ; T)-f_{\mathrm{R}}(E ; T)\right]
$$

where $f_{\mathrm{L} / \mathrm{R}}(E ; T)=\left(\exp \left(\left[\mu_{\mathrm{L} / \mathrm{R}}-E\right) / T\right]+1\right)^{-1}$, and where we ignore the energy dependence of $\Gamma_{\mathrm{L} / \mathrm{R}}$. At $T=0$, and in the absence of interactions, this current (7) reduces to our previous transistor-current estimate (3). Scattering introduces important density-of-state changes, $\delta N_{\text {orb }}(E)$. Nevertheless, transistor robustness follows because this DOS change must integrate to zero (electron-number conservation): assuming the bias condition (5), the nonequilibrium current integral (7) always includes most of this spectral weight when 
the transistor is enabled (4). In particular, conservation rules ensure a robust quantummechanical transistor operation at room temperature, $T \approx 25 \mathrm{meV}$, when $e V_{\text {bias }} \gtrsim 6 T \sim$ $150 \mathrm{meV}$ (Fig. 2).

In summary, we have proposed and documented theoretically a robust nanoscaletransistor effect in a resonant-tunneling nanotube heterostructure. Such double-junction nanotube transistor structures should be possible to produce ${ }^{18}$ and to be probed, like the current-rectifying single-junction nanotube diode. ${ }^{14}$ We have detailed how the fundamental conservation laws guarantee the important device robustness, i.e. insensitivity towards both scattering and finite-temperature operation. We have, for a specific realization, presented a quantitative materials characterization and detailed study of the predicted device operation. We stress that a range of nanotube heterostructures can realize the predicted transistor effect.

Acknowledgements. We thank John W. Wilkins and Henrik Rydberg for highly valued comments and discussions. This work was supported by the Swedish Foundation for Strategic Research (SSF) through the Materials Consortium no. 9. 


\section{REFERENCES}

${ }^{1}$ G. E. Moore, Electronics Apr. 19, 114 (1965).

${ }^{2}$ R. P. Feynman, Engineering and Science Feb. 1960, 22 (1960).

${ }^{3}$ S. Iijima, P. M. Ajayan, and T. Ichihashi, Phys. Rev. Lett. 69, 3100 (1992).

${ }^{4}$ C. Dekker, Phys Today May 1999, 22 (1999).

${ }^{5}$ J. W. Mintmire, B. T. Dunlap, and C. T. White, Phys. Rev. Lett. 68, 631 (1992).

${ }^{6}$ N. Hamada, S.-I. Sawada, and A. Oshiyama, Phys. Rev. Lett. 68, 1579 (1992).

${ }^{7}$ S. J. Tans, A. R. M. Vershueren, and C. Dekker, Nature 393, 49 (1998).

${ }^{8}$ C. Weisbuch, and B. Vinter, Quantum Semiconductor Structures (Academic Press, Inc., Boston, 1991), p. 142.

${ }^{9}$ R. Tsu, and L. Esaki, Appl. Phys. Lett. 22, 562 (1973).

${ }^{10}$ G. Baym, and L. P. Kadanoff, Phys. Rev. 124, 287 (1961); D. C. Langreth, "1975 NATO Advanced Study Institute on Linear and Nonlinear Electron Transport in Solids, Antwerpen, 1975," Vol. B17 (Plenum, New York, 1976), pp. 3-32,

${ }^{11}$ S. Hershfield, J. H. Davies, and J. W. Wilkins, Phys. Rev. Lett. 67, 3720 (1991).

${ }^{12}$ P. Hyldgaard, S. Hershfield, J. H. Davies, and J. W. Wilkins, Annals of Physics 236, 1 (1994).

${ }^{13}$ R. Saito, G. Dresselhaus, and M. S. Dresselhaus, Phys. Rev. B 53, 2044 (1996).

${ }^{14}$ Y. Yao, H. W. Ch. Postma, L. Balents, and C. Dekker, Nature 402, 273 (1999).

${ }^{15}$ C. T. White, J. W. and Mintmire, Nature 394, 30 (1998).

${ }^{16}$ E. Merzbacher, Quantum Mechanics (John Wiley \& Sons, New York, 1970), pp. 124-133.

17 J. Bardeen, Phys. Rev. Lett. 6, 57 (1961). 
${ }^{18}$ Y. Yao, H. W. Ch. Postma, L. Balents, and C. Dekker, Nature 402, 276 (1999): Supplement to Ref. 14.

${ }^{19}$ D. Orlikowski, M. B. Nardelli, J. Bernholc, and C. Roland, Phys. Rev. Lett. 83, 4132 (1999).

${ }^{20}$ S. J. Tans, M. H. Devoret, R. J. A. Groeneveld, and C. Dekker, Nature 394, 761 (1998).

${ }^{21}$ L. Chico, M. P. L. Sancho, and M. C. Munoz, Phys. Rev. Lett. 81, 1278 (1998).

${ }^{22}$ P. Hyldgaard and J. W. Wilkins, Phys. Rev. B 53, 6889 (1996).

${ }^{23}$ A. Zangwill, "Physics at Surfaces" (Cambridge University Press, Cambridge, 1988), pp. $163-170$.

${ }^{24}$ E. Hult, H. Rydberg, B. I. Lundqvist, and D. C. Langreth, Phys. Rev. B 59, 4708 (1990); H. Rydberg, B. I. Lundqvist, D. C. Langreth, and M. Dion, Phys. Rev. B, in print.

${ }^{25}$ P. Hyldgaard, unpublished.

${ }^{26}$ M. Büttiker, Phys. Rev. Lett. 57 (1986), 1761. 


\section{FIGURES}

FIG. 1. Sample nanotube current-switch and transistor realization. A short, $L_{B} \approx 3 \mathrm{~nm}$, section of nonconducting fullerene barrier (central honeycomb region) separates a pair of surrounding metallic nanowires (grey horizontal tubes), which form leads to the source and drain contacts (top triangles). The molecular barrier traps a lowest resonance orbital $\Psi_{\text {orb }}$, left insert, with energy position $E_{\text {orb }}$ controlled by a close metal gate (bottom triangle) at voltage $\Phi_{\text {gate }}$. The right insert illustrates the transistor-gate control: At $\Phi_{\text {gate }} \sim 0$, the molecular gap $E_{g}$ (dashed box) inhibits transport as the resonant orbital (dashed line) are forced far above the chemical potentials; However, a voltage, $\Phi_{\text {gate }} \sim 2 \mathrm{~V}$ adjusts the molecular potential (solid curve) to a new minimum, suppresses $E_{\text {orb }}$ (solid line), and permits a resonant-tunneling load current (drain-to-source) $J<J_{\mathrm{RT}} \sim 5 \mu \mathrm{A}$. The (base-to-source) input current remains vanishing, $J_{\text {in }}<J_{\mathrm{V}} \ll 1 \mathrm{nA}$.

FIG. 2. Robust nanosized current-switch and transistor effects. Both operations are documented at a fixed applied bias $e V_{\text {bias }}=\mu_{\mathrm{L}}-\mu_{\mathrm{R}}$, chosen at six times $\Gamma \equiv \Gamma_{\mathrm{L}}+\Gamma_{\mathrm{R}}$. A robust current-switch effect (top and insert panel) arises as the gate voltage increases $\mu_{\mathrm{L}}-E_{\text {orb }}$ and enable ('on') the load current. The top panel documents device robustness by contrasting the load-current estimate (2) (solid curve) against current estimates at a finite temperature $T=\Gamma$ (dotted curve), and in the presence of a strong nonequilibrium scattering (dashed curve): a phonon mode of energy $\Omega_{0}=3 \Gamma$. The insert panel details the implicit gate control, $E_{\text {orb }}^{(*)}\left(\Phi_{\mathrm{B}}\right)-\mu_{\mathrm{L}}$, for the lowest two well-resolved resonance energies and validates our single-resonance transport model. The pair of dashed curves identifies the zone $\left|\mu_{\mathrm{L}}-E_{\text {orb }}\right|<6 \Gamma$ (=eV bias) in which we document the current-swich and transistor operation; This zone lies well within the level separation and transport is thus focused onto the lowest resonance orbital $E_{\text {orb }}$ with a scattering-insensitive nonequilibrium transport mechanism. We predict a robust nanosized transistor effect (bottom panel), $J \propto J_{\text {in }}$, which is characterized by a large amplification: $J_{\mathrm{RT}} / J_{\mathrm{V}}>10^{3}$. 


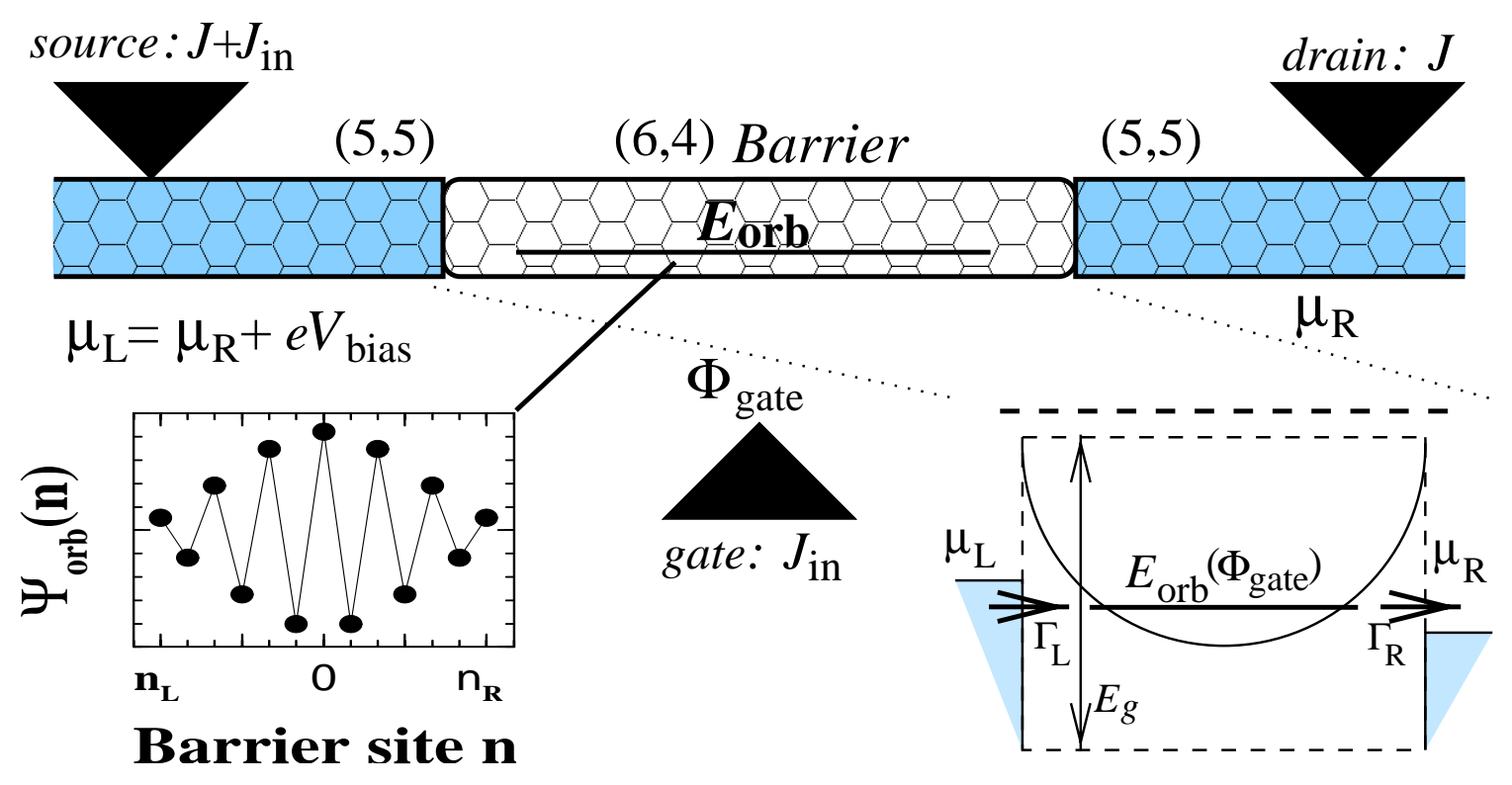

Figure 1. Double-column format. PER HYLDGAARD, Chalmers University of Technology. Robust nanosized transistor effect in fullerene-tube heterostructure. 


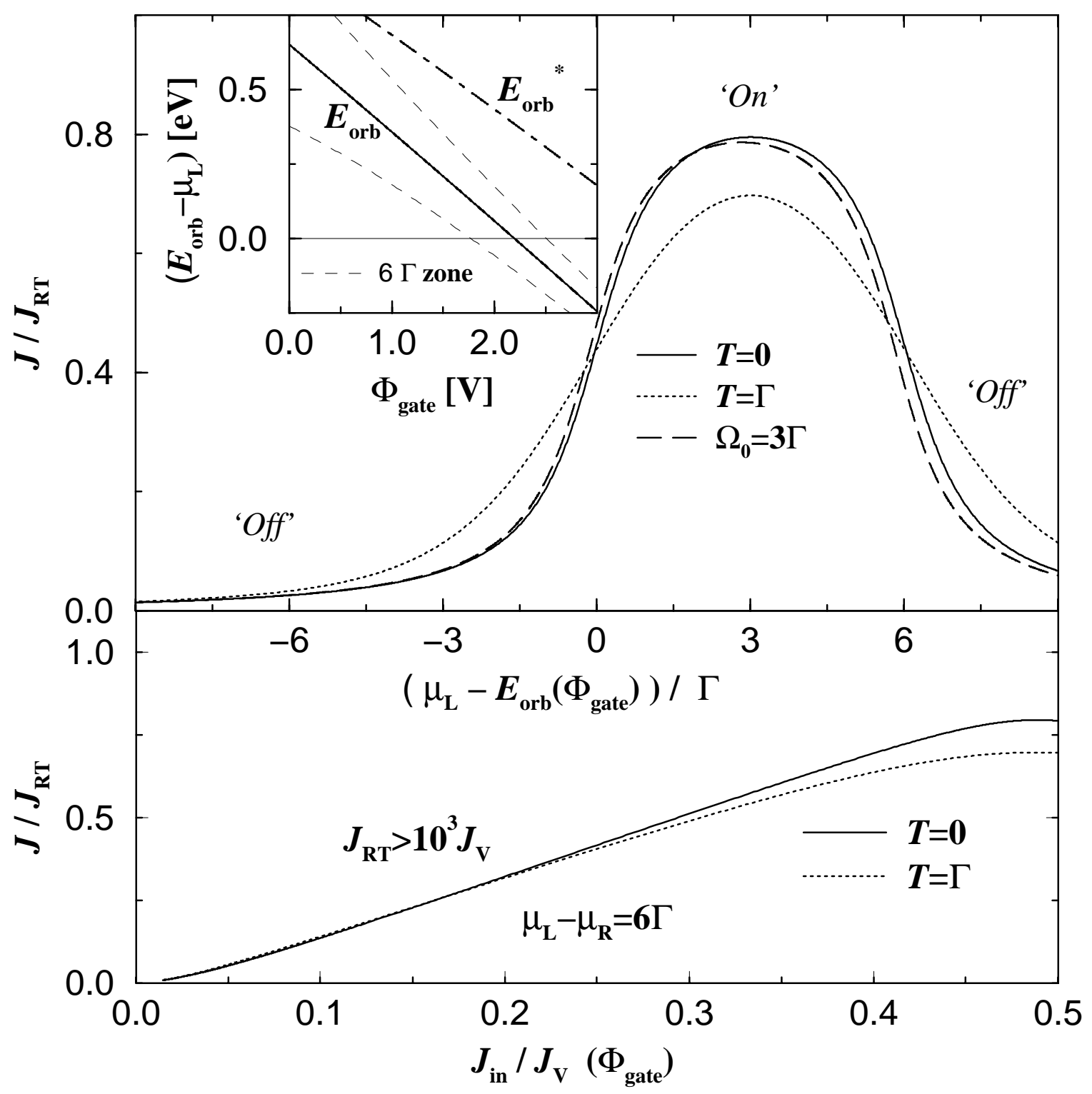

Figure 2. Fits one-column format. PER HYLDGAARD, Chalmers University of Technology. Robust nanosized transistor effect in fullerene-tube heterostructure. 